(1)

CrossMark

\title{
The value of chest CT as a COVID-19 screening tool in children
}

To the Editor:

It is difficult to identify children infected with coronavirus disease 2019 (COVID-19) who have little or no respiratory symptoms. For routine clinical care in different circumstances, it is relevant to assess the COVID-19 status of patients. Routine PCR is recognised as the gold standard but can be falsely negative due to sampling errors. For diagnosing and monitoring adult COVID-19 patients, characteristic radiological lesions have been recognised $[1,2]$ and to assess the possibility of COVID-19 infection in adults scheduled for surgery in whom a PCR test is negative or missing, a non-enhanced chest computed tomography (CT) scan has been proposed as an option in the Netherlands [3] because: 1) patients may be pre-symptomatic in the incubation period of COVID-19 infection and subsequently develop symptoms post-operatively, implying a greater risk for adverse post-operative outcomes; and 2) patients may be asymptomatic or mildly symptomatic carriers and shedders of COVID-19, and place hospital workers and other patients at risk.

It is unknown whether CT scanning has additional value as a screening tool to rule out COVID-19 infections in children with little or no respiratory symptoms or with negative or missing PCR test results. It would require very convincing evidence to justify the introduction of ionising radiation to rule out COVID-19 infection in children.

Therefore, we conducted a literature search to assess what is known about the sensitivity, specificity and negative predictive value of chest CT as a screening tool to rule out COVID-19 infections in children. We searched for PubMed articles in English or with English abstracts, including mesh terms COVID and CT scan and [adults or children] published up to 29 April 2020. This resulted in 92 papers that mostly focused on the use of CT scans in diagnosing and/or monitoring the disease or its severity [1,2] rather than ruling it out. Most studies did not include subjects with negative PCR, so the true negative rate or specificity of CT scanning could not be calculated; instead, we focused on sensitivity, the ability of chest $\mathrm{CT}$ to recognise as many patients as possible.

From several case series in PCR-proven adult COVID-19 patients with little or minor symptoms, it is clear that chest CT abnormalities may be absent in spite of mild symptoms, with a sensitivity varying between $44 \%$ [4], 69\% [5] and 70\% [6], depending on the study.

In one observational study, sensitivity was exceptionally high (97\%). In this case series of 1014 clearly symptomatic hospitalised patients from the highly endemic Wuhan (China) area, the large majority of patients had abnormal CT scans, resulting in a sensitivity of $97 \%$ but with a specificity of $25 \%$, implying a high false-positive rate [7]. From that study it was unclear at what moment in the course of disease diagnostic tests were conducted. Chest CT in more advanced disease is associated with more abnormalities [4], and this is especially the case in selected clinical populations [8]. In those series, the positive and negative predictive values of the CT scan were $65 \%$ and $83 \%$, respectively. In another study in a highly epidemic region, $82 \%$ of patients with mild symptoms had chest CT abnormalities [9]. Such results may only be valid in epidemic areas with high pre-test probability for this disease, and one should not assume similar sensitivity of chest CT in a general population for screening purposes $[7,8]$

With a prevalence of disease of $\sim 60 \%$ as in Wuhan, the positive and negative predictive values were $65 \%$ and $83 \%$, respectively. However, in an area with a lower or higher prevalence the positive and negative predictive values will differ (figure 1).

In summary, in a considerable percentage of adult COVID-19 patients with little or no symptoms, a chest CT may be normal. Depending on the study population, the sensitivity of a chest CT to detect abnormalities in proven COVID-19 patients ranged 44-97\% (median 69\%). 
FIGURE 1 Based on the Wuhan (China) data from adult patients [7], the positive predicted value (PPV] and negative predictive value (NPV) of chest CT can be calculated. This figure illustrates that PPV and NPV highly depend on the prevalence of disease in the study population. SARS-CoV-2: severe acute respiratory syndrome coronavirus 2 .

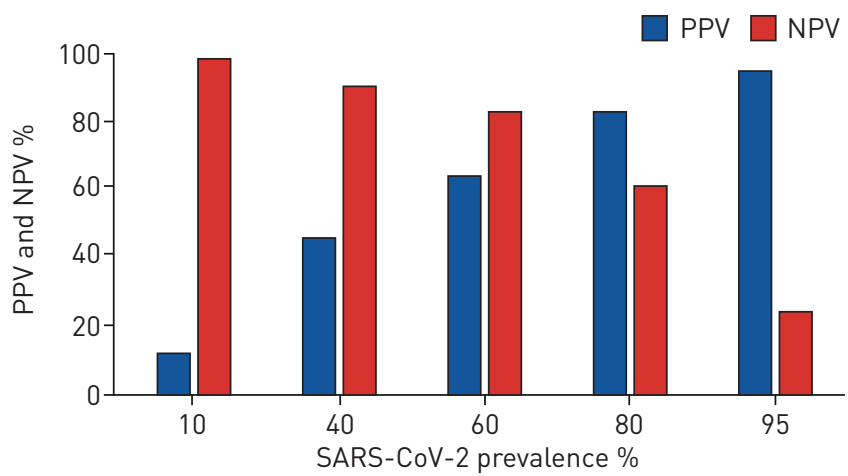

In children, the course of the disease in general is milder than in adults. Both symptomatic and asymptomatic children with known contact with persons having confirmed or suspected COVID-19 infection $(n=1391)$ were evaluated in a Chinese cohort [10]. COVID-19 was confirmed through PCR in $171(12.3 \%)$ out of 1391 children. Amongst these 171, CT abnormalities consistent with (any) viral pneumonia were found in 111, implying a sensitivity of 65\%. In 27 (16\%) out of 171 infected children, there were no symptoms or abnormalities on CT images and in 33 (19\%) infected children, only an upper respiratory tract infection was observed. Hence, in $\sim 35 \%$ of proven paediatric COVID-19 patients, CT images were normal. In a retrospective study on clinical data and chest CT of 15 children diagnosed with COVID-19 infection, 10 of whom were asymptomatic, six (40\%) showed no lesions on chest CT [11]. In a case series of five young COVID-19-positive children, four were asymptomatic; of these, 50\% had some minor abnormalities on CT scan and 50\% had normal CT images [12]. Of 13 hospitalised children with mild-to-severe COVID-19, six (46\%) had no abnormalities on CT scan [13]; out of 115 paediatric clinical patients infected with COVID-19, 24\% had no abnormalities on CT scan [14].

In summary, in paediatric COVID-19-positive patients with little or only minor upper airway symptoms, a chest CT may be normal in 35-50\% of cases. Hence, depending on the study, the reported sensitivities of CT scanning to detect abnormalities in paediatric COVID-19 patients range 50-74\% (median 60\%).

A screening test should accurately identify diseased and non-diseased individuals. An ideal screening test is affordable, safe and patient-friendly, highly sensitive (high probability of detecting disease) and highly specific (high probability that those without the disease will screen negative) with high positive and negative predictive values.

Studies in asymptomatic adults and children are scarce. In adult studies, chest CT can be normal in a considerable percentage of symptomatic COVID-19 patients. The median (range) sensitivity of finding chest CT abnormalities was 69\% (44-97\%) in COVID-19-proven (mildly) symptomatic adult patients. According to five paediatric studies, normal chest CT has been reported in a large percentage of children with asymptomatic or mild COVID-19 disease. The median (range) sensitivity of finding CT image abnormalities was only $60 \%(40-74 \%)$ in COVID-19-proven paediatric patients.

This implies an unacceptable percentage of false-negative cases, creating a false sense of safety for healthcare personnel while exposing the child to potentially harmful ionising radiation, that will not contribute to a better outcome for the child.

CT scanning should always be carefully considered in children due to the potential harm that ionising radiation may cause [15]. A routine chest CT for viral pneumonia in children is rarely performed, except in cases with unexplained symptoms or complications that could require a change in therapy.

With the information currently available and considering the PCR as gold standard for the diagnosis COVID-19, a negative chest CT scan does not rule out COVID-19 lung disease in at least a third of the paediatric COVID-19 patients who tested positive and who have little or no respiratory symptoms. Therefore, we consider CT scanning of the chest to be unsuitable as a screening tool to rule out COVID-19 in paediatric patients with little or no symptoms.

Peter J.F.M. Merkus ${ }^{1}$ and Willemijn M. Klein ${ }^{2}$

${ }^{1}$ Dept of Paediatrics, Division of Respiratory Medicine and Allergology Radboud University Medical Centre, Amalia Children's Hospital, Nijmegen, The Netherlands. ${ }^{2}$ Dept of Radiology and Nuclear Medicine, Radboud University Medical Center, Amalia Children's Hospital, Nijmegen, The Netherlands. 
Received: 17 April 2020 | Accepted after revision: 29 April 2020

Conflict of interest: P.J.F.M. Merkus has nothing to disclose. W.M. Klein has nothing to disclose.

\section{References}

1 COVID-19 CORADS classification: COVID working group of the Dutch Radiological Society. https:// radiologyassistant.nl/chest/covid-19-corads-classification. Date last accessed: 4 June 2020. Date last updated: 3 March 2020.

2 ACR recommendations for the use of chest radiography and computed tomography (CT) for suspected COVID-19 infection. https://www.acr.org/Advocacy-and-Economics/ACR-Position-Statements/Recommendations-for-ChestRadiography- and-CT-for-Suspected-COVID19-Infection. Date last accessed: 4 June 2020 date last updated 22 March 2020.

3 Practice Guideline: Preoperative Work-up for COVID-19 Infection in Asymptomatic Patients Scheduled for Surgery Under General Anesthesia. https://heelkunde.nl/sites/heelkunde.nl/files/Practice\%20Guideline\%20Preoperative\%20work \%20up\%20on\%20possible\%20COVID-19\%20infection\%20in\%20asymptomatic\%20patients.pdf Date last accessed: 4 June 2020. Date last updated: 2 April 2020.

4 Bernheim A, Mei X, Huang M, et al. Chest CT findings in coronavirus disease-19 (COVID 19): relationship to duration of infection. Radiology 2020; 295: 200463.

5 Li K, Fang Y, Li W, et al. CT image visual quantitative evaluation and clinical classification of coronavirus disease (COVID-19). Eur Radiol 2020; in press [https://doi.org/10.1007/s00330-020-06817-6].

$6 \mathrm{Hu}$ Z, Song C, Xu C, et al. Clinical characteristics of 24 asymptomatic infections with COVID 19 screened among close contacts in Nanjing, China. Sci China Life Sci 2020; 63: 706-711.

7 Ai T, Yang Z, Hou H, et al. Correlation of chest CT and RT-PCR testing in coronavirus disease 2019 (COVID 19) in China: a report of 1014 cases. Radiology 2020; in press [https://doi.org/10.1148/radiol.2020200642].

8 Wáng YXJ. A call for caution in extrapolating chest CT sensitivity for COVID 19 derived from hospital data to patients among general population. Quant Imaging Med Surg 2020; 10: 798-799.

9 Guan WJ, Ni ZY, Hu Y, et al. Clinical characteristics of coronavirus disease 2019 in China. N Engl J Med 2020; 382: $1708-1720$.

10 Lu X, Zhang L, Du H, et al. SARS-CoV-2 enfection in children. N Engl J Med 2020; 382: 1663-1665.

11 Feng K, Yun YX, Wang XF, et al. Analysis of CT features of 15 children with 2019 novel coronavirus infection. Zhonghua Er Ke Za Zhi 2020; 58: 275-278.

$12 \mathrm{Li} \mathrm{W}$, Cui H, Li K, et al. Chest computed tomography in children with COVID 19 respiratory infection. Pediatr Radiol 2020; 50: 796-799.

13 Tan X, Huang J, Zhao F, et al. [Clinical features of children with ARS-CoV-2 infection: an analysis of 13 cases from Changsha, China]. Zhongguo Dang Dai Er Ke Za Zhi 2020; 22: 294-298.

14 Ma YL, Xia SY, Wang M, et al. [Clinical features of children with SARS-CoV-2 infection: an analysis of 115 cases]. Zhongguo Dang Dai Er Ke Za Zhi 2020; 22: 290-293.

15 Meulepas JM, Ronckers CM, Smets AMJB, et al. Radiation exposure from pediatric CT scans and subsequent cancer risk in the Netherlands. J Natl Cancer Inst 2019; 111: 256-263. 